\title{
Experimental and numerical analysis of mechanical behaviour of AISI 316L austenitic stainless steel: two level homogenization - neutron diffraction
}

\author{
Jamal Fajoui ${ }^{1, a^{*}}$, David Gloaguen ${ }^{1, b}$, Vincent Legrand ${ }^{1, \mathrm{c}}$, Guy Oum ${ }^{1, \mathrm{~d}}$, \\ Joe Kelleher ${ }^{2, e}$, Winfried Kockelmann ${ }^{2, f}$
}

${ }^{1}$ Université de Nantes, Centrale Nantes, Institut de Recherche en Génie Civil et Mécanique (UMR CNRS 6183), 58, rue Michel Ange - BP 420, 44606 Saint-Nazaire Cedex, France

${ }^{2}$ ISIS Facility, Science and Technology Facilities Council, Rutherford Appleton Laboratory, Harwell Oxford Didcot, Oxfordshire, UK OX11 0QX

a*: jamal.fajoui@univ-nantes.fr, b. david.gloaguen@univ-nantes.fr,

c: vincent.legrand@univ-nantes.fr, ${ }^{\text {. }}$ : guy.oum@univ-nantes.fr, e: joe.kelleher@stfc.ac.uk, f: winfried.kockelmann@stfc.ac.uk

Keywords: mechanical behavior, plasticity, dislocation, neutron diffraction, homogenization, selfconsistent model, Kröner approach

\begin{abstract}
A two-level homogenization approach is adopted in order to predict the stress state of deformed polycrystals in correlation with the microstructure. This study is devoted to complex loading paths behaviour of cubic material. A mechanical description of the grain is developed through a micro-meso transition based on elastoplastic Kröner's model. The meso-macro transition using a self-consistent approach is applied to deduce the global behaviour. Mechanical tests and neutron diffraction measurements are used to validate and assess the model.
\end{abstract}

\section{Introduction}

Technological evolution and competitiveness require the development of systems more complex with benefit. In this context, the control of metallic materials properties is essential to develop any structure. The manufacturing processes shaping plates are limited by several phenomena like forming fold-line, shear bands, cracks, cavities, etc. These phenomena are relatively easy to study in monotonic loadings and become very difficult to predict during strain-path changes.

Classic one transition level models were developed to predict the influence of the local mechanical behavior to the global one. The grain is considered as an uniform region neglecting the formation of the dislocation microstructure. These approaches permit to predict correctly the yield surface, the crystallographic texture linked to the plastic deformation, the intergranular stresses due to initial and induced anisotropy during monotonic loadings. Nevertheless, this kind of description fails to reproduce the mechanical behaviour during changing strain paths. These methods do not, or weakly, take account of the formation, the evolution and the stability of induced dislocation microstructures which are strongly path dependent.

In this work, a two-level homogenization approach is developed for the micromechanical modelling of the elastoplastic material behavior [1]. This study is particulary devoted to complex loading paths. At the microscopic scale, the mechanical behavior is described by the Kröner model. The grain is considered as two-phase material: dislocations walls (with high density dislocation) and cells (with low density dislocation). The intragarnular heterogeneities are highlighted by a nonlocal work-hardening, which is linked to the two-phase description. At the global scale, the mechanical behavior is deduced from the mesoscopic one by an elastoplastic self-consistent model. To validate our numerical approach and explain the Bauschinger mechanisms, we conclude this paper by a confrontation between the simulation results with those from neutron diffraction (ND). This will allow to get a complete analysis at different levels: diffraction volume scale and macroscopic one. 


\section{Experimental data}

The material used in the present work is a standard 316L austenitic steel alloy. Samples were tested using the ENGIN-X beamline at the ISIS facility, Rutherford Appleton Laboratory (Didcot, UK) [2], using a $50 \mathrm{kN}$ Instron testing machine. Briefly, the ENGIN-X instrument presents a horizontal loading axis positioned at $45^{\circ}$ to the incident beam. Two detector banks are set up horizontally and at angles $\pm 90^{\circ}$ to the incident beam, allowing simultaneous measurements of lattice strains in both parallel (loading direction denoted LD) and perpendicular (transverse direction, TD) directions to the applied load, in the opposing $90^{\circ}$ detector banks. The time-of-flight technique enables collection of an entire diffraction pattern (effective d-spacing range from 0.88 to $2.63 \AA$ ) in each detector simultaneously, owing to the specificity of the neutron beam. The detector banks cover $\pm 14^{\circ}$ in the horizontal plane and $\pm 21^{\circ}$ in the vertical plane [2]. The gauge volume was $4 \mathrm{x} 4 \mathrm{x} 4 \mathrm{~mm}^{3}$, and the recording time was taken over $15 \mathrm{~min}$ for each measurement at a given macroscopic strain. Macroscopic strain was monitored on the sample using a clip gauge. Single peak fitting was performed using the Open Genie code [3]. Tension-compression tests, were performed at room temperature. We consider two pre-strains: 2 and $6 \%$ in tension mode for the first test, and, $-2 \%$ and $-3 \%$ in compression mode for the second one. In situ diffraction volume analyses were performed in order to follow the evolution of intergranular strains during complex loading (tension-compression) at different strain rates with an interval of $0.5 \%$. Experimental results were obtained for several reflections: (111), (200), (220), (311), (331), (420), (422) and (511). For a more detailed description of ND, see Ref. [4].

\section{Two-level homogenization model}

We consider single-phase polycrystals such as f.c.c. metals. Based on the work of Mughrabi [5], each grain is treated as a two-phase composite consisting of cell walls of high dislocation density and cell interiors relatively poor in dislocations. The phases have the same isotropic elastic behavior described by shear modulus $\mu$ and Poisson's ratio $v$. The grain undergoes a strain $\varepsilon^{\text {II }}$ (with a stress $\left.\sigma^{\mathrm{II}}\right)$ composed of an elastic part $\varepsilon^{\mathrm{e}}$ and a plastic part $\varepsilon^{\mathrm{p}}$. Describing by $\varepsilon^{\mathrm{pc}}$ and $\varepsilon^{\mathrm{pw}}$ the mean plastic strain in the dislocation cells (c) and walls (w) respectively, one can write:

$$
\varepsilon^{I I}=(1-f) \varepsilon^{w}+f \varepsilon^{c}
$$

where $f_{c}$ (respectively $f_{w}=1-f_{c}$ ) is the volume fraction of cell interiors (respectively cell walls) in a grain. Using the same mixture rule for stress, one has:

$$
\sigma^{I I}=(1-f) \sigma^{w}+f \sigma^{c}
$$

$\sigma^{\alpha}$ corresponds to the stress tensor in the phase $\alpha$ (the superscript ' $\alpha$ ' stands for walls ' $w$ ' and cells 'c') and $\sigma^{\mathrm{II}}$ is the stress tensor induced in the grain.

With an isotropic elastic behaviour:

$$
\varepsilon^{p I I}=(1-f) \varepsilon^{p w}+f \varepsilon^{p c}
$$

The plastic strain rate for each phase is the following:

$$
\dot{\varepsilon}^{p \alpha}=\sum_{g} R_{\alpha}^{g} \dot{\gamma}_{\alpha}^{g}
$$

where $R_{\alpha}^{g}$ is the Schmid tensor on a system g. $\dot{\gamma}_{\alpha}^{g}$ denotes the slip rate on a deformation system $\mathrm{g}$.

The elastic strain rate is linked to the stress rate in the grain through the Hooke's law:

$$
\dot{\varepsilon}^{\text {ell }}=\dot{\varepsilon}^{I I}-\dot{\varepsilon}^{p I I}=s: \dot{\sigma}^{I I}
$$

where $\mathrm{s}$ is the elastic compliance tensor at the grain level. A:B denotes the double scalar product $\mathrm{A}_{\mathrm{ijk} l} \mathrm{~B}_{\mathrm{klmn}}$ using the Einstein summation convention.

The main mechanism responsible of the hardening involves dislocations that generated inside grains and built a particular architecture. These structures could be viewed as composites consisting of hard dislocation walls having high local dislocation density, separated by soft regions (cells) having 
low local dislocation density. Few dislocations accumulate within cells. The annihilation of dislocations is mainly localized inside walls due to the rearrangement of dislocations associated with the plastic strain inside walls. Based on these physical processes, the rate of increase of dislocations density inside the walls on a slip system $\mathrm{g}$ is:

$$
\dot{\rho}_{w}^{g}=\dot{\rho}_{w}^{g+}+\dot{\rho}_{w}^{g-} \text { with: } \dot{\rho}_{w}^{g+}=\frac{1}{b L^{g}} \dot{\gamma}_{c}^{g}, \dot{\rho}_{w}^{g-}=-\frac{2 y_{c}}{b} \rho_{w}^{g} \dot{\gamma}_{w}^{g} \text { and } L^{g}=\frac{k_{L}}{\sqrt{\sum_{h \neq g} \rho_{w}^{h}}}
$$

$\dot{\rho}_{w}^{g+}$ corresponds to the rate of increase of dislocation density inside the walls on the system g. This term is responsible for the creation and the development of the cell structure. This flux consists of dislocations that glide towards walls through the applied stress.

$\dot{\rho}_{w}^{g-}$ is the annihilation term which takes into account the dynamic recovery during the deformation.

$\mathrm{b}$ is the magnitude of the Burgers vector and $\mathrm{y}_{\mathrm{c}}$ is proportional to the annihilation distance of dislocation dipoles. $\mathrm{L}^{\mathrm{g}}$ is the mean free path of mobile dislocations in the cell interiors associated to the deformation system g. Its evolution is governed by the density of point obstacles (essentially cell walls) cutting the system $g$ and creating interactions with gliding dislocations.

The critical resolved shear stress (CRSS) can be related to the dislocations densities by the hardening relation:

$$
\tau_{c \alpha}^{g}=\tau_{c 0 \alpha}^{g}+\xi \mu b \sqrt{\sum_{h} a^{g h} \rho_{\alpha}^{h}}
$$

$\tau_{c 0 \alpha}^{g}$ is the initial reference shear stress on system $\mathrm{g}$ and $\xi$ is a constant depending on the interaction of dislocations $(\xi \in[0.25-0.6])$. agh is a hardening matrix which terms depend on the type of interactions between dislocation families $g$ and h. Only two terms, $a_{g=h}^{g h}$ and $a_{g \neq h}^{g h}$ (respective selfand latent hardening parameters), will be considered. $\rho_{\alpha}^{h}$ is the dislocation density in the phase $\alpha$. During plastic strain, dislocations, generated inside cell, moves and stores in walls. Hence, the cell structure affects the walls. This is the non-local hardening concept. In the general case, the nonlocal hardening laws can be written for a two-phase composite as follows [6]:

$$
\dot{\tau}_{c c}^{g}=\sum_{h} H_{c c}^{g h} \dot{\gamma}_{c}^{h}+\sum_{h} H_{c w}^{g h} \dot{\gamma}_{w}^{h} \text { and } \dot{\tau}_{c w}^{g}=\sum_{h} H_{w c}^{g h} \dot{\gamma}_{c}^{h}+\sum_{h} H_{w w}^{g h} \dot{\gamma}_{w}^{h}
$$

$H_{c c}^{g h}, H_{w w}^{g h}, H_{c w}^{g h}$ and $H_{w c}^{g h}$ are the hardening matrices describing interactions between dislocation moving on various slip systems. $H_{c c}^{g h}$ and $H_{w w}^{g h}$ interpret the influence of system $\mathrm{h}$ on $\mathrm{g}$ once in the same phase. $H_{c w}^{g h}$ and $H_{w c}^{g h}$ stand the non-local hardening and the influence of a given phase on the other one. For an appropriate representation of the hardening behaviour, these matrices are expressed with microstructural variables such as dislocation density. By combining the previous equations (8), the work-hardening matrices $H_{w w}^{g h}$ and $H_{w c}^{g h}$ are:

$$
H_{w w}^{g h}=-\frac{y_{c} \xi^{2} \mu^{2} b}{\left(\tau_{c w}^{g}-\tau_{c o w}^{g}\right)} \rho_{w}^{h} a^{g h} \text { and } H_{w c}^{g h}=\frac{\xi^{2} \mu^{2} b}{2\left(\tau_{c w}^{g}-\tau_{c o w}^{g}\right)} \frac{a^{g h}}{L^{h}}
$$

Because of the low accumulation of dislocations inside cells, $H_{c c}^{g h}$ is simply described by a constant. The influence of walls on the evolution of dislocations in cells can be considered as negligible. Finally:

$$
H_{c c}^{g h}=\frac{\varsigma}{\tau_{c w}^{g}-\tau_{c o w}^{g}} a^{g h} \text { and } H_{c w}^{g h}=0
$$

During the plastic regime, the variation of the cell interior volume fraction for a grain I is considered as a function of the accumulated slip through the following empiric relation [6]:

$$
f_{c I}=f_{\infty}+\left(f_{0}-f_{\infty}\right) \exp \left(-\frac{\gamma_{c I}^{a c c}}{\gamma^{p a r}}\right)
$$

where $f_{0}$ is the initial volume fraction and $f_{\infty}$ the saturation value at large strains. $\gamma^{\text {par }}$ is a parameter which describes the decrease rate of $\mathrm{f}_{\mathrm{cl}} . \gamma_{c I}^{a c c}$ is the accumulated slip in the cell in the grain I . This relation allows to take the intergranular plastic heterogeneity into account. The volume fraction of 
cells for each crystallite develops differently, in relation to the effect of the grain orientation. It only depends on the plasticity activity. In the remainder of the paper, the cell volume fraction will be denoted $\mathrm{f}_{\mathrm{c}}$ instead of $\mathrm{f}_{\mathrm{c} I}$.

Kröner [7] was based on the Eshelby's works to write the following relation between two-phases and grain states:

$$
\sigma^{\alpha}=\sigma^{I I}+\alpha_{T} c^{\alpha}:\left(I-s^{e s h}\right):\left(\varepsilon^{p w}-f \varepsilon^{p \alpha}\right)
$$

I is the fourth order identity tensor. $c^{\alpha}$ is the elastic constant tensor for the phase $\alpha . s^{\text {esh }}$ is the Eshelby tensor expressing interaction of an inclusion with a given morphology independently of its elastic properties. The tensor $s^{\text {esh }}$ is evaluated based on elastic properties of the matrix and the mechanical predicted behaviour will be too stiff. One may retain the convenience of Kröner's treatment but make a useful assumption of plastic accommodation. An accommodation factor $\alpha_{\mathrm{T}}$ is introduced. This crude but simple and efficient approximation permits to simulate quite well the mechanical response of real metals [6]. This factor has been used in the sequel with an additional assumption concerning $\alpha_{\mathrm{T}}$. This factor is supposed to be independent of the plastic flow and $\alpha_{\mathrm{T}}=$ $1 / 10$. In the remainder of the paper and for more clarity, the factor $\alpha_{T}$ is omitted.

Taking into account the relations (1) and (2), the mean stress tensors $\sigma^{\mathrm{c}}$ and $\sigma^{\mathrm{w}}$ within the cells and the walls can be written as:

$$
\begin{aligned}
& \sigma^{c}=\sigma^{I I}+(1-f) c^{c}:\left(I-s^{e s h}\right):\left(\varepsilon^{p w}-f \varepsilon^{p c}\right) \\
& \sigma^{w}=\sigma^{I I}+f c^{w}:\left(I-s^{e s h}\right):\left(\varepsilon^{p w}-f \varepsilon^{p c}\right)
\end{aligned}
$$

The plastic flow can take place in a grain when the Schmid criterion is verified, i.e. slip occurs if the resolved shear stress $\tau_{\alpha}^{g}$ on a system $\mathrm{g}$, for each phase, is equal to the critical value $\tau_{c \alpha}^{g}$ depending on the hardening state of the slip system. This necessary condition is insufficient, and the complementary condition, which states that the increment of the resolved shear stress must be equal to the incremental rate of the critical resolved shear stresses (CRSS), has to be checked simultaneously. The resolved shear stress is defined as the projection of the phases stress tensor $\sigma^{\alpha}$ on the considered deformation system. In small strain formulation, one has:

$$
\tau_{\alpha}^{g}=R_{\alpha}^{g}: \sigma^{\alpha}=\tau_{\alpha}^{g} \text { and } \dot{\tau}_{\alpha}^{g}=R_{\alpha}^{g}: \dot{\sigma}^{\alpha}=\dot{\tau}_{\alpha}^{g}
$$

We used a new formulation to resolve the problem of ambiguous selection of deformation systems and reduce the running time of the computations $[1,6]$. The slip rate can be expressed by the following equation:

$$
\dot{\gamma}_{\alpha}^{g}=K_{\alpha}^{g} \dot{\tau}_{\alpha}^{g}
$$

The slip rates $\left(\dot{\gamma}_{c}^{g}\right.$ and $\left.\dot{\gamma}_{w}^{g}\right)$ are linked to the resolved shear stress rates $\left(\dot{\tau}_{c}^{g}\right.$ and $\left.\dot{\tau}_{w}^{g}\right)$ through a Hyperbolic tangent function $K_{\alpha}^{g}$. This function depends on the $\tau_{c \alpha}^{g}$ and $\tau_{\alpha}^{g}$. Hence, it's able to describe the hardening behavior during the plastic regime. By developing (1) - (17), one obtains after calculations the following matrix form:

$$
\dot{\gamma}_{c}^{g}=T_{c}^{g}: \dot{\sigma}^{I I} \text { and } \dot{\gamma}_{w}^{g}=T_{c}^{g}: \dot{\sigma}^{I I}
$$

After some mathematics development, the linear relation is obtained:

$$
\dot{\varepsilon}^{I I}=\left[s+\sum_{g}\left(f R_{c}^{g}: T_{c}^{g}+(1-f) R_{w}^{g}: T_{w}^{g}\right)\right] . . \dot{\sigma}^{I I}=\ell^{-1}: \dot{\sigma}^{I I}
$$

$\ell^{-1}$ is the microscopic tangent moduli tensors. This tensor depends on active systems, elastic properties, stress rate and deformation history of each phase.

To deduce the macroscopic behavior from de microscopic one, we used the EPSC model. For a more detailed description of the EPSC model, see Refs. [1,6]. 


\section{Comparison between experiment and simulation}

This section aims to analyze predictions of the proposed model. Especially, the estimation of local and global behavior of austenitic stainless steel during complex load path at different strain rate.

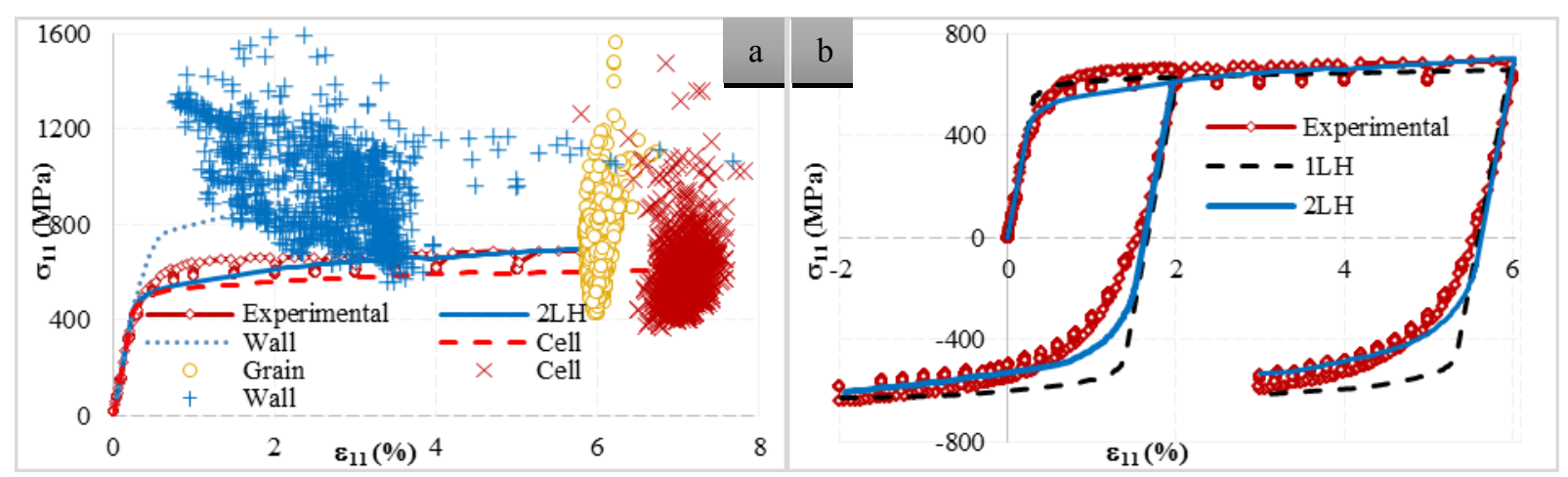

Fig. 1. a: Experimental curve and simulated pre-strain loading curve. Scattered points represent the local strains and internal stresses heterogeneity. b. Comparison between experimental and simulated stress-strain curves during complex load path

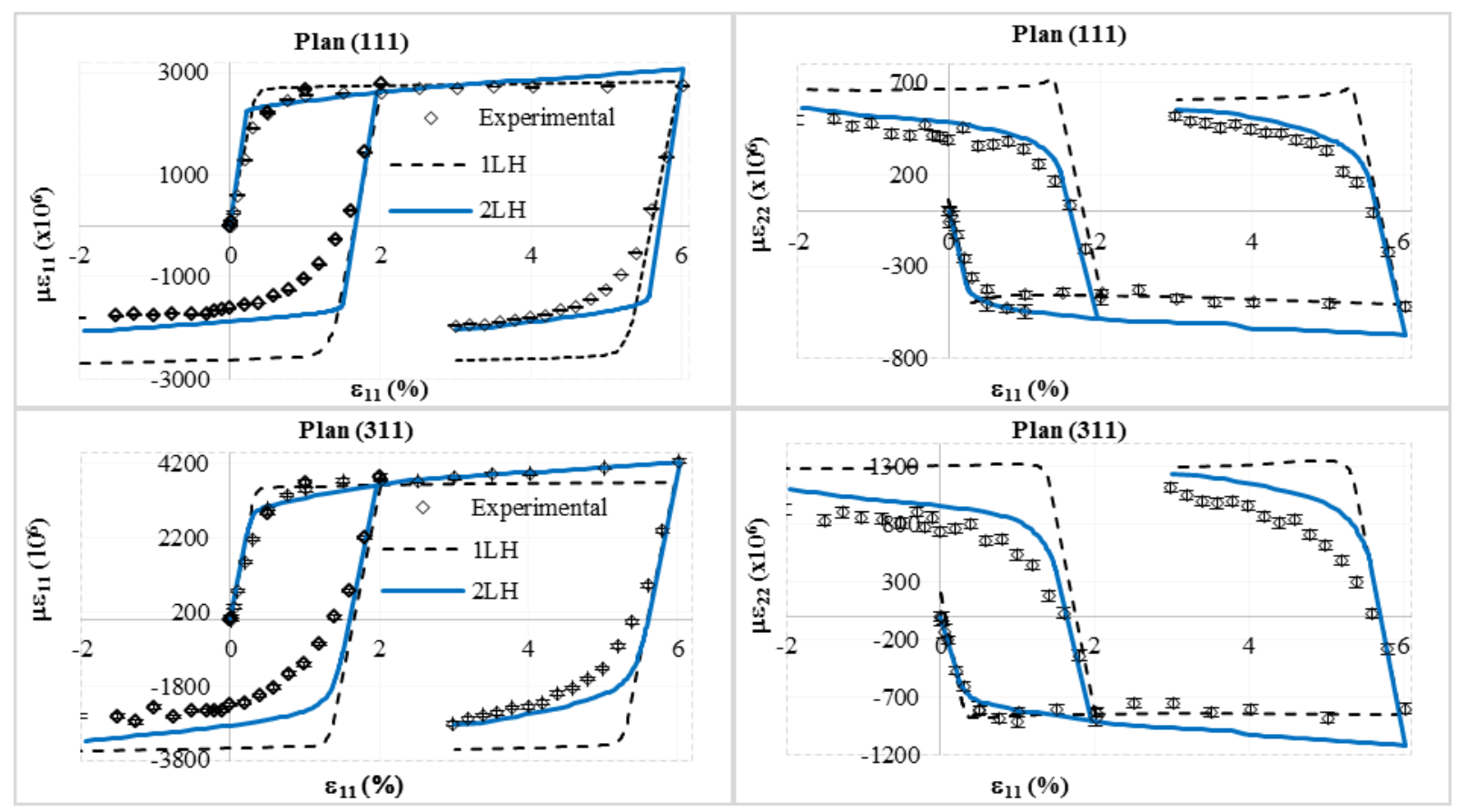

Fig. 2: Experimental and simulated results for diffracting volume behavior during sequential loading tests

In order to perform numerical simulations, the model parameters were identified by fitting the experimental points corresponding to the macroscopic curve of monotonic loading. The elastic properties are considered as isotropic $(\mu=70 \mathrm{GPa}$ and $v=0.3)$. Texture measurements were performed with the GEM beamline at the ISIS facility. The texture is described by a weighted discrete distribution of orientations (Euler angles $\left.\left(\varphi_{1}, \phi, \varphi_{2}\right)\right)$. The model calculations were performed for a set of 1000 spherical inclusions. This number of crystallites has been chosen to simulate the polycrystalline aggregate in an efficient way. For f.c.c. metals, the plastic deformation was modelled assuming $\{110\}<111>$ slip systems.

In Fig. 1.a., the stress-strain curve obtained with the present model (2LH) and the experimental one for the first loading are compared. A good correlation is obtained with the reference data. Fig. 1.a. also shows the internal mechanical states within cells and walls: $\sigma_{11}^{\alpha}=\sum_{l} f_{I} \sigma_{11}^{I \alpha}$ and $\varepsilon_{11}^{\alpha}=\sum_{I} f_{I} \varepsilon_{11}^{I \alpha}$. where $\mathrm{f}_{\mathrm{I}}$ represents the volume fraction of grain I. The two phases don't reach their yield limit at the same time. The dislocation density within walls is much higher than the cells one. 
The material becomes heterogeneous since the wall phase is generated. The induced microstructure appears with the plastic strain. The Fig. 1.a shows simultaneously the third-order stresses $\sigma_{11}^{I \alpha}$ for each phase and the resulting mesoscopic stresses $\sigma_{11}^{I}$ at the grain level just before and after the elastic unloading.

Fig. 1.b. shows experimental and predicted results for the tension-compression tests at two prestrains: $2 \%$ and $6 \%$ for $2 \mathrm{LH}$ and the Elastoplastic Self-Consistent model (EPSC: "1 Level Homogenization" 1LH). It can observed that the present model captures all the essential features (reloading yield stress, macroscopic work hardening) associated with the Bauschinger effect. The EPSC scheme fails to explain and reproduce correctly the softening effects during the compression loading. We can see also that the elastic limit is larger for tension in comparison with compression. In author's opinion, this difference seems to be due that the initial residual stresses in our sample (not virgin) which are very important. These stresses will disappear in the first loading (plastic slip). Later, they influence the lattice strains for tensile and compression.

Fig. 2. gives the local behavior of two lattice planes (111) and (311) along the longitudinal and transversal directions during complex load path. For the sake of clarity, only two planes were presented. Generally, the simulated results show that the major features in experimental stress-strain curves can be captured for the first and the second loading. Therefore, the Bauschinger effect is predicted in diffracting volume scale. One observe that the EPSC don't reproduce the mechanical behavior of the volume diffracting during the compression loading. The classical EPSC scheme fails to explain and reproduce correctly the softening effects during the compression loading. This method weakly takes into account the formation, the evolution and the stability of induced dislocations microstructures which are strongly path dependent.

\section{Conclusions}

In this paper, a new approach was developed to predict the elasto-plastic behaviour of polycrystalline aggregates under sequential loadings. This approach is based on a two-level transitions. The grain is considered as a two-phase material with cell walls (with high density dislocation) and cell interiors (with low dislocation density). A mechanical description of the grain is developed through a micro-meso transition based on a modified Kröner approach. The intragranular heterogeneities are highlighted by a non-local work-hardening, which is linked to the two-phase description. Correct agreement is observed between the simulation data and the experimental results (neutron diffraction and mechanical tests) at volume diffracting and macroscopic levels.

\section{References}

[1] D. Gloaguen, G. Oum, V. Legrand, J. Fajoui, S. Branchu, Experimental and theoretical studies of intergranular strain in an alpha titanium alloy during plastic deformation, A. Materialia, 61, 15, (2013), 5779-5790.

[2] J. R. Santisteban, M.R. Daymond, J.A. James, L. Edwards, ENGIN-X: a third-generation neutron strain scanner, J. A. Crystallography, 39, (2006), 812-825.

[3] C. M. Moreton-Smith, S. D. Johnston, F.A. Akeroyd, Open-GENIE, a generic, multi-platform program for the analysis of neutron scattering data, J. N. Research, 4, (1996), 41-47.

[4] W. Kockelmann, L. C. Chapon, P. G. Radelli, Neutron texture analysis on GEM at ISIS, Physica B, 385, (2006), 639-643.

[5] H. Mughrabi, The long-range internal stress field in the dislocation wall structure of persistent slip bands, P. S. Solidi (a) 104, (1987), 107-120.

[6] J. Fajoui, D. Gloaguen, B. Courant, R. Guillén, Micromechanical modelling of the elastoplastic behaviour of metallic material under strain-path changes, C. Mechanics, 44, (2009), 285-296.

[7] E. Kröner, On the plastic deformation of polycrystals. A. Metallurgica, 9, (1961), 155-161.

[8] P. Franciosi, The concepts of latent hardening and strain hardening in meallic single crystals, Acta Metall., 33 (1985), 1601-1612. 